\title{
Optimization of Digital Image Correlation for High-Resolution Strain Mapping of Ceramic Composites
}

\author{
V.P. Rajan • M.N. Rossol · F.W. Zok
}

Received: 15 August 2011 / Accepted: 2 April 2012 / Published online: 14 April 2012

(C) Society for Experimental Mechanics 2012

\begin{abstract}
Digital image correlation (DIC) is assessed as a tool for measuring strains with high spatial resolution in woven-fiber ceramic matrix composites. Using results of mechanical tests on aluminum alloy specimens in various geometric configurations, guidelines are provided for selecting DIC test parameters to maximize the extent of correlation and to minimize errors in displacements and strains. The latter error is shown to be exacerbated by the presence of strain gradients. In a case study, the resulting guidelines are applied to the measurement of strain fields in a $\mathrm{SiC} / \mathrm{SiC}$ composite comprising 2-D woven fiber. Sub-fiber tow resolution of strain and low strain error are achieved. The fiber weave architecture is seen to exert a significant influence over strain heterogeneity within the composite. Moreover, strain concentrations at tow crossovers lead to the formation of macroscopic cracks in adjacent longitudinal tows. Such cracks initially grow stably, subject to increasing app lied stress, but ultimately lead to composite rupture. Once cracking is evident, the composite response is couched in terms of displacements, since the computed strains lack physical meaning in the vicinity of cracks. DIC is used to identify the locations of these cracks (via displacement discontinuities) and to measure the crack opening displacement profiles as a function of applied stress.
\end{abstract}

V.P. Rajan · M.N. Rossol · F.W. Zok (ه)

Materials Department, University of California,

Santa Barbara, CA 93106-5050, USA

e-mail: zok@engineering.ucsb.edu
Keywords 3-D digital image correlation - Woven-fiber ceramic-matrix composites - Spatial resolution • Strain error $\cdot$ Crack opening displacement

\section{Introduction}

Digital image correlation is a non-contact optical technique used to measure surface displacements and strain fields of a test specimen subjected to an external stimulus (load, temperature, etc.) [1]. Displacements are obtained by imaging a speckle pattern on the specimen surface during the test and subsequently correlating each image of the deformed pattern to that in the undeformed state [2]. Strains are obtained by differentiating displacement fields. The technique combines sub-pixel displacement accuracy [3] with excellent spatial resolution, allowing strains to be measured to within $10^{-4}$ or less [4]. In principle, these attributes make DIC eminently suitable for probing strain distributions in woven ceramic fiber/ceramic matrix composites. Conventional measurement techniques (e.g. strain gages and extensometers) are often inadequate for three principal reasons.

First, strain distributions in woven fiber composites can be highly heterogeneous and dependent on the weave architecture [5]. The local strains in a fiber tow are influenced by numerous factors, including: the orientation of the tow with respect to the loading axis; the amplitude of out-of-plane tow undulation; the local 'environment' around the tow, characterized by the arrangement of neighboring tows and matrix; and the elastic/fracture properties of the composite constituents. Furthermore, the strains are expected to 
vary spatially over a length scale comparable to the tow dimensions. To formulate high-fidelity models of composite damage and failure, it is necessary to find the maximum strain values at the tow level. The implication is that strains must be resolved spatially at a length scale smaller than that of the tows.

Second, strain variations arise in the presence of structural features such as holes or notches. In these cases, the gauge length for strain measurement must be less than the smallest characteristic dimension of the structural feature. Strain gauges and extensometers lack the requisite spatial resolution to meet these requirements in most cases of practical interest.

Third, the mechanical behavior of ceramic composites must be understood at temperatures representative of the targeted service conditions $\left(1200-1500^{\circ} \mathrm{C}\right)$. Although contacting extensometers can be used to obtain global strains, only non-contact methods are viable for measuring strain variations in this domain. Indeed, imaging capabilities needed to perform DIC measurements at elevated temperatures have recently been established and their use demonstrated on woven $\mathrm{C} / \mathrm{SiC}$ composites [6]. While the analyses of displacement and strain errors described later in this paper remain relevant, additional experimental challenges and measuring artifacts, including illumination, heat haze and speckle pattern degradation, arise at elevated temperatures.

Numerous studies have utilized digital image correlation to understand the mechanical behavior of fiber composites [7-13]. Two recent studies [14, 15] have incorporated DIC to examine the mechanical behavior of a 2-D C-fiber polymer matrix composite. They confirm the expected features: notably, the heterogeneity of strains at intra-tow and inter-tow length scales as well as the periodicity of strains and its intimate relationship to the fiber architecture. They also demonstrate the suitability of DIC for measuring local strains with high accuracy and spatial resolution.

Two additional important issues have been raised in these and other papers. First, there are inherent trade-offs between spatial resolution and displacement accuracy. Second, there is complex interplay between displacement error, strain error, and the parameters chosen for the speckle pattern, image correlation and strain calculation $[1,3,4,16-20]$. Although several studies have attempted to quantify these relationships, most have been limited to correlation errors arising from rigid body translations $[17,21]$ or no motion at all [4]. Simulated experiments in which displacements are applied numerically to a computer-generated image have also been conducted [3, 18, 19, 22]. The latter results represent best-case scenarios since they neglect experimental errors caused by camera vibration, imperfect image contrast, etc.

The present study represents part of a broad research activity focused on strain evolution, damage and rupture of woven ceramic composites. The overarching objective of the present study is to establish a framework for design and analysis of DIC experiments that yield high-fidelity strain measurements in these materials. This is accomplished by: (i) identifying and analyzing sources of displacement and strain error in mechanical tests that produce uniform strains as well as strain gradients; (ii) formulating analytic relationships between these errors and applied strain, speckle size, and DIC algorithm parameters; and (iii) utilizing the results to design experiments to probe strain variations in a ceramic composite with the requisite spatial resolution.

The outline of the paper is as follows. First, the principles of digital image correlation are reviewed. Next, test results on a baseline (homogeneous) material (Al 6061-T6) are analyzed to determine the relationships between displacement and strain error and the DIC algorithm parameters. Three specimen geometries are considered: one with nominally uniform strain (uniaxial tension) and two with strain gradients (open-hole and center-notched tension). Because the latter geometries yield different strain gradients as well as different length scales for strain variation, they provide useful insights and guidance on the feasibility of measuring strain gradients and small-scale strain heterogeneities in woven composites. This part of the study complements previous work focused on errors arising from rigid body motions $[4,17,21]$. Finally, a case study illustrates how the preceding analyses can be used to design tests on a $\mathrm{SiC} / \mathrm{SiC}$ composite. In addition to revealing the effects of fiber architecture on strain distributions, the tests are used to probe the initiation and growth of cracks. To this end, displacement fields found via digital image correlation are analyzed to locate cracks and measure their opening displacements. Such measurements are crucial for calibrating models of crack bridging in fiber composites.

\section{Error Analysis}

\section{Digital Image Correlation}

In-plane surface displacements are measured by tracking the deformation of a speckle pattern through a series of digital images acquired during a mechanical test. If the out-of-plane displacements of the sample are negligible, a single (stationary) camera can be used. Otherwise, to measure out-of-plane displacements and 
to correctly extract in-plane displacements, stereo images (from two cameras) must be employed. The correlation algorithm attempts to locate a subset of the image of the undeformed pattern corresponding to a subset in the image of the deformed pattern. In mathematical terms, it seeks to find [2]:

$\underset{\mathbf{p}}{\arg \min } \sum_{\mathbf{x} \in \text { Subset }}(G(\xi(\mathbf{x}, \mathbf{p}))-F(\mathbf{x}))^{2}$

where $G$ and $F$ are the grayscale intensity values of the deformed and undeformed subsets, respectively; $\xi$ is the shape function that describes the deformation between the subsets; and $\mathbf{p}$ comprises the parameters of the subset shape function, chosen to minimize the sum of squared differences (SSD) between grayscale intensity values in the two corresponding subsets [2]. The DIC software used in this work (Vic-3D, Correlated Solutions [23]) provides the option of using more complex correlation schemes; these include center-weighting of the sum in equation (1) and accounting for changes in intensity values between images (offsets and scaling) [23]. The minimization process results in the assignment of a displacement vector $(u, v, w)$ to the subset center. Correlation is subsequently repeated throughout the sample to generate a displacement field over a rectangular array of subset centers, defined as nodal points.

There are several critical parameters for correlation. One is the subset size, $h_{\text {sub }}$ (in pixels). As Bornert et al. [18] have convincingly demonstrated, the spatial resolution of displacement measurement is governed predominantly by the subset size; sinusoidal displacement fields of a wavelength less than $h_{\text {sub }}$ cannot be measured. The lower bound on subset size is set by the requirement that each subset contain unique information to distinguish it from neighboring subsets. The rule of thumb adopted in the DIC literature to satisfy this requirement is that $h_{\mathrm{sub}}>3 \cdot h_{\mathrm{sp}}$, where $h_{\mathrm{sp}}$ is the speckle size [2]. Furthermore, to avoid aliasing, each speckle must contain several pixels. The corresponding rule of thumb is $h_{\mathrm{sp}}>3$ pixels [2]. Conversely, large speckles necessitate large subset sizes, thereby reducing spatial resolution.

The order of the subset shape function also plays an important role. For an $n$ th-order shape function, displacement errors scale with the $n+1$ th derivative of displacement [18]; the shape function employed by Vic-3D is first order (affine) with an additional term to account for camera perspective. The requirement that second-order derivatives be small (to minimize displacement error) sets an upper bound on the allowable subset size.
When displacement fields are numerically differentiated (in the simplest case, by finite differences), the resulting strain fields are usually 'noisy' [24]. The strain fields are therefore filtered by Gaussian-weighted averaging over a prescribed $N \times N$ array of nodal points. The distance between nodal points is defined as the step size, $h_{\mathrm{st}}$ (in pixels), and the gage length over which strain averaging is performed is defined as the filter length, $h_{f}$ (in pixels) (clearly, $h_{f}=N \cdot h_{\mathrm{st}}$ ). The effects of $h_{\text {sub }}, h_{\mathrm{st}}$, and $h_{f}$ on displacement and strain error both in uniform strain fields and under large strain gradients are addressed below.

\section{Experimental Details}

\section{Sample preparation}

In this part of the study, tests were performed on $1.5 \mathrm{~mm}$ thick sheets of aluminum 6061-T6. Three test configurations were employed: uniaxial tension, openhole tension, and center-notched tension. Samples were machined via electro-discharge machining. The uniaxial tension test sample had dog-bone geometry with a gauge section of $250 \mathrm{~mm}$ long and $25 \mathrm{~mm}$ wide. Both the open-hole tension and center-notched tension specimens were machined from rectangular strips, $300 \mathrm{~mm}$ long and $50 \mathrm{~mm}$ wide. The open-hole diameter was $12 \mathrm{~mm}$, and center-notch was $12 \mathrm{~mm}$ in length with a $0.6 \mathrm{~mm}$ tip radius. Since the length scale for strain decay in the vicinity of such features is controlled by the root radius, the strain gradient in the center-notched sample is expected to persist over a length scale 1/10th of that in the open-hole test.

Two speckling techniques were employed. In both, samples were first coated with flat white spray paint. Speckles were subsequently applied using either a spray canister with flat black spray paint or a Paasche airbrush with black water-soluble paint.

\section{Mechanical testing and DIC setup}

All samples were tested at room temperature on a hydraulic testing machine (MTS 810, Minneapolis, MN) at a nominal strain rate of $10^{-4} / \mathrm{s}$. Samples were clamped with hydraulic grips. Strains on the back-face of the uniaxial tension specimen were measured using a laser extensometer (Electronic Instrument Research, Irwin, PA) over a gauge length of $25 \mathrm{~mm}$.

Images for DIC were taken with a pair of digital cameras (Point Grey Research Grasshopper), each with a CCD resolution of $2448 \times 2048$ pixels and a 70-180 mm lens (Nikon ED AF Micro Nikkor). The 
focal length of the lenses was $70 \mathrm{~mm}$, the aperture setting was F-16, and the angle between cameras was $24^{\circ}$. For all experiments described in this work, either the maximum or the minimum focal length was employed to minimize errors arising from differences in magnification between the two cameras. For the three aluminum alloy specimens, images were taken at similar magnifications: 41 pixels $/ \mathrm{mm}$ for the open-hole and center-notched tension tests and 36 pixels $/ \mathrm{mm}$ for the uniaxial tension test. The area of the open-hole and center-notched specimens within the field of view was $50 \mathrm{~mm} \times 50 \mathrm{~mm}$, while that for the uniaxial tension specimen was $50 \mathrm{~mm} \times 25 \mathrm{~mm}$. The area of interest for image correlation was selected to exclude un-speckled regions such as the hole or notch.

\section{Speckle pattern characterization}

Two approaches are employed for quantifying the speckle size distribution. One is an autocorrelation approach (AC), described by Rubin [25] and used in previous DIC studies $[2,17,18]$. The average speckle size, $h_{\mathrm{sp}}$, is the width of the autocorrelation function, calculated from the points satisfying the equation $A(u)=$ 0.5 , where $A$ is the autocorrelation function [2]. The other approach relies on particle analysis (PA) techniques [26]. These have been implemented in various software packages; the present analysis is performed using ImageJ [27]. Prior to analysis, the camera image is thresholded and converted to a binary image. Contiguous features of any size and circularity are found within the new image. The effective diameter of each feature (speckle) is defined as $d_{\mathrm{eq}}=4 \cdot A / P$, where $A$ is its area and $P$ is its perimeter. Using this approach, circular speckles yield an effective diameter equal to the actual diameter; for elliptical speckles, the effective diameter is the geometric mean of the major and minor axes of the ellipse.

\section{Computation of error}

In DIC displacement analyses, three types of errors are typically reported: the bias, $\overline{\Delta v}$, the standard deviation, $v_{\mathrm{SD}}$, and the root-mean square error, $v_{\mathrm{RMS}}[17,18]$. The bias is a measure of the systematic deviation of the measured displacements, $v_{m}$, from their true (imposed) values, $v_{i}$, given by

$$
\overline{\Delta v}=\frac{1}{n} \sum_{j=1}^{n}\left(v_{m}-v_{i}\right)
$$

Because the displacement bias is typically negligible compared to the random error, characterized by the standard deviation [18], $v_{\mathrm{SD}} \approx v_{\mathrm{RMS}}$, where

$v_{\mathrm{RMS}}=\sqrt{\frac{1}{n} \sum_{j=1}^{n}\left(v_{m}-v_{i}\right)^{2}}$

Only the RMS error is considered in this work. Strain errors are computed in an analogous manner, with displacements, $v$, replaced by $\epsilon_{y y}$ ( $y$ denoting the nominal loading direction). Here, again, bias is typically much smaller than the corresponding standard deviation and thus only the RMS strain error is considered.

The imposed displacement field in the uniaxial tensile test is unknown a priori. It is taken to be of the form

$v=\epsilon_{i} y+C_{1} x+C_{2}$

where $x$ is the transverse in-plane direction; $\epsilon_{i}$ is the imposed axial strain (taken to be that measured by a 'virtual extensometer' over a gage length of $40 \mathrm{~mm}$ on the sample surface); $C_{1}$ represents the displacement gradient $\partial v / \partial x$ associated with rigid body rotations (small but not negligible); and $C_{2}$ is the rigid body translation (a natural consequence of applying extension to only one end of the test specimen). $C_{1}$ and $C_{2}$ are determined by fitting equation (4) to the measured (DIC) displacement data. In this scheme, the displacement bias is assumed to be zero, and therefore the RMS displacement error computed using equation (3) is solely a measure of the random error. The sum in equation (3) is evaluated over a sufficiently large array of nodal points to ensure statistically significant results. The RMS strain error is computed in a similar manner.

In the open-hole and center-notched tension tests, only strain error is determined. Axial normal strain values $\left(\epsilon_{y y}\right)$ from the DIC software are taken along a line emanating from the edge of the hole or the notch at the sample mid-plane along the x-direction. Strains are interpolated between nodal points using cubic splines. The true spatial variation of axial strain along this line was obtained from finite element analysis, which utilized a material constitutive law calibrated with the uniaxial tensile data. (Although the law was calibrated for both elastic and plastic deformation, the subsequent measurements were restricted to the elastic domain only). The strains obtained from DIC and FEA along with equation (3) are used to calculate the RMS error. 
Results and Discussion

\section{Speckle size}

Speckle size distributions for both the airbrush and spray paint patterns and the relevant statistical values are summarized in Fig. 1. The figure indicates that the speckles from the airbrush pattern are finer than that from the spray paint pattern. Furthermore, both speckle size distributions are highly skew, implying the presence of speckles several times larger than the median value. The skewness can be problematic for correlation; indeed, correlation is difficult to attain in regions of the pattern where the speckle size is large. These observations suggest that the median speckle size is not the premier characteristic of a speckle size distribution, since it is relatively insensitive to the presence of very large speckles. Statistics which account for skewness, such as the autocorrelation speckle size (which is implicitly weighted by the number of pixels within a speckle) or the 90th percentile speckle size (from PA) should be utilized instead. Hereafter, $h_{\mathrm{sp}}$ is taken to be that obtained from the autocorrelation method. (Numerically, the values obtained from AC are almost the same as the 90th percentile values.)

\section{Correlation}

Full image correlation requires that each subset in the deformed image contain sufficient unique information so that it can be located in the undeformed image [18]. This requirement sets a lower bound on the allowable subset size. Figure 2(a) illustrates the effects of subset

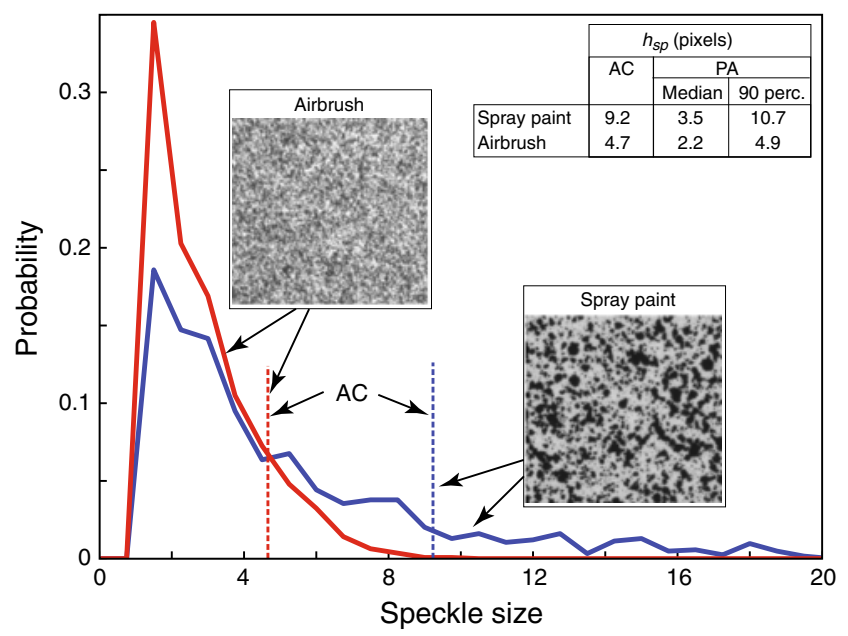

Fig. 1 Speckle size distribution from particle analysis (PA, solid lines) and autocorrelation (AC, dashed lines)

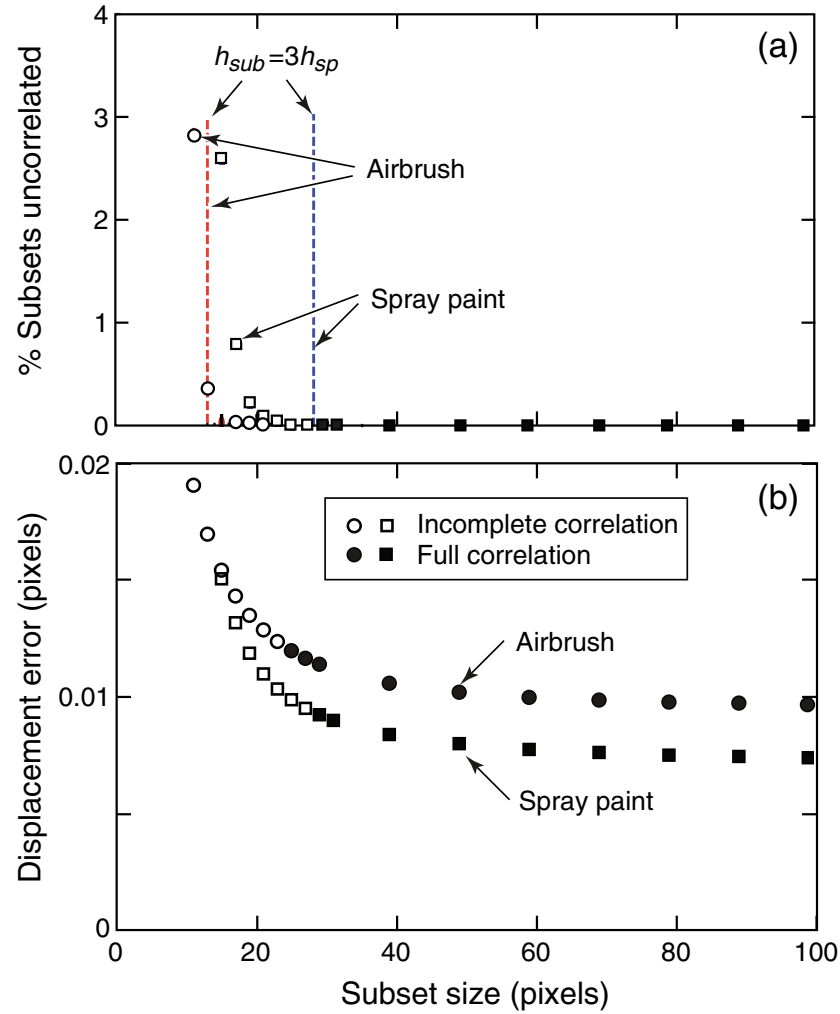

Fig. 2 Variation in (a) extent of correlation and (b) RMS displacement error with subset size for uniaxial tension tests on Al. The predicted subset for full correlation from the rule $h_{\text {sub }}>$ $3 \cdot h_{\mathrm{sp}}$ is shown by dashed lines in (a)

size (below and above this bound) on the degree of correlation (based on error criteria prescribed by the correlation algorithm). The results are broadly consistent with the rules of thumb for achieving optimal correlation: $h_{\mathrm{sp}}>3$ pixels and $h_{\mathrm{sub}}>3 \cdot h_{\mathrm{sp}}$ [2]. The latter describes the qualitative trend of Fig. 2(a) - that larger speckle sizes require larger subset sizes for full correlation. The minimum subset size predicted by the rule of thumb and the subset size required for full correlation also agree quantitatively. For the spray painted speckles, $h_{\mathrm{sp}}=9.3$ pixels and hence the minimum recommended subset size is $h_{\text {sub }}=28$ pixels. By comparison, the experimental results show that $99 \%$ correlation is obtained at 17 pixels and full $(100 \%)$ correlation at 29 pixels. For the airbrushed speckles, $h_{\mathrm{sp}}=4.5$ pixels and the predicted lower bound on subset size is 13 pixels. $99 \%$ correlation is attained at a subset size of 13 pixels and full correlation at 25 pixels.

\section{Displacement error}

Figure 2(b) shows the effect of subset size on displacement error for the uniaxial tension test at an 
applied strain of 0.002 (about half of the yield strain and thus well within the elastic domain). For small subset sizes, below that needed for full correlation, the error scales approximately inversely with subset size. In contrast, for larger subsets, the error asymptotes to a constant value. Similar trends have been reported in previous studies $[17,21]$. The displacement errors obtained in the present study $(0.01-0.02$ pixels $)$ also agree with the results of other studies [17, 28]. The subset size that provides the best compromise between spatial resolution and displacement accuracy lies just below that at the asymptote; larger subset sizes yield no further reduction in displacement error. The nearoptimal value employed in the subsequent strain error analysis is taken to be 40 pixels for the spray paint pattern. Note that, in general, the optimal subset size depends on a number of factors, including the speckle size distribution and presence of strain gradients within the subset.

For subset shape functions that are first-order, displacement error is expected to be insensitive to the magnitudes of rigid body displacements and/or uniform strains within the subset [18]. This hypothesis can be assessed for the case of uniform strain; the results are shown in Fig. 3. For strains below yielding (i.e. $<0.004$ ) displacement error is indeed constant. Furthermore, the error for low strains is identical to that for nominally zero applied strain. This quantity was computed by taking the standard deviation of displacements between two sequential images (before loading). Interestingly, for strains in excess of the yield strain, the error increases dramatically. It is surmised that the latter trend is a consequence of the inherent heterogeneity of crystal plasticity at the length scales being probed by the present measurements $\left(h_{p} / 10 \approx 3 \mu \mathrm{m}\right)$.

\section{Strain error}

The sources of strain error in digital image correlation are twofold. First, as mentioned previously, numerical differentiation of 'noisy' displacement data results in 'noisy' strain data [24]. Filtering (averaging) is therefore employed. However, filtering can also introduce strain error if derivatives of strain within the filtering gage length are not negligible. In the uniaxial tension test, the strains are uniform and thus only the former error source is relevant; for the open-hole and centernotched tension tests, both sources must be considered.

The relationship between strain error, displacement error, and subset size for the uniaxial tension test at an applied strain of 0.002 is depicted in Fig. 3. Here, $h_{\mathrm{st}}=2$ pixels and $h_{f}=10$ pixels: both small in comparison to the selected subset sizes. Because displace-
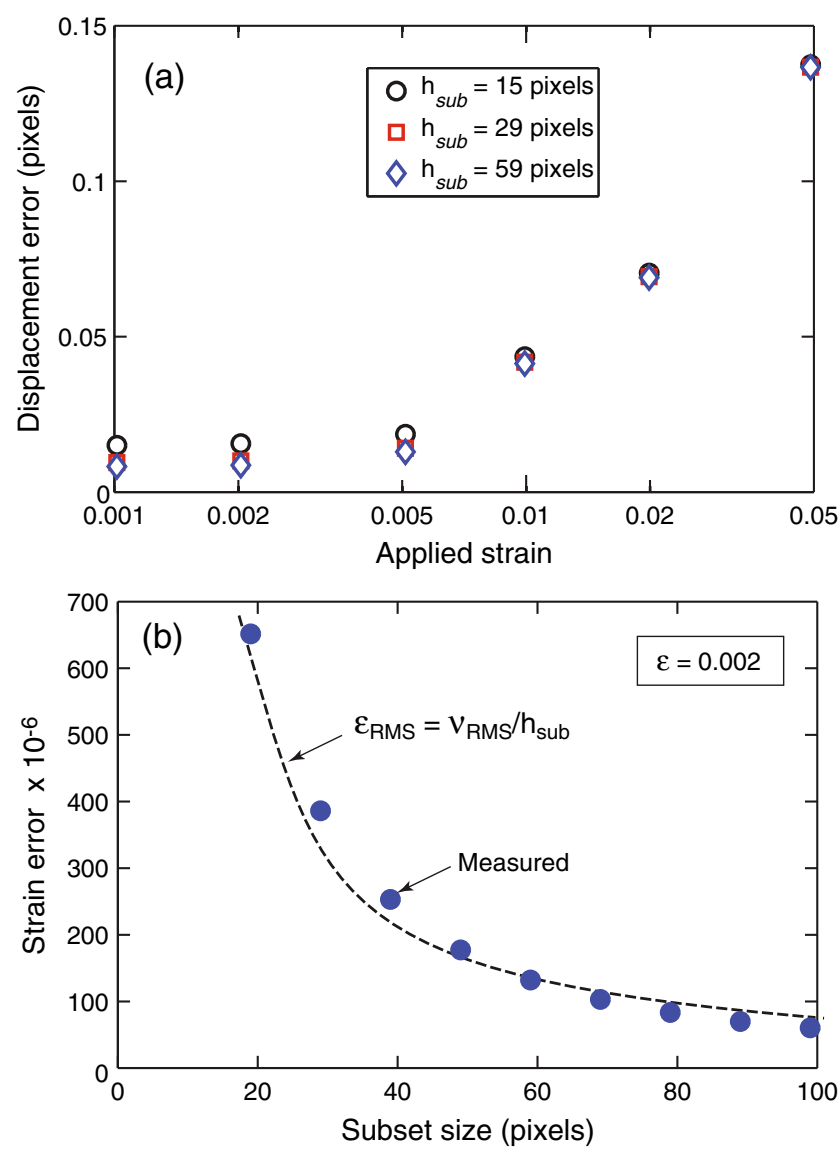

Fig. 3 Effects of strain and subset size on displacement and strain errors $\left(h_{\mathrm{st}}=2\right.$ pixels, $h_{f}=10$ pixels $)$

ment values within a subset are correlated to one another, displacement data exhibit sinusoidal variations with a wavelength roughly equal to the subset size. Hence, differentiation of the displacement field to obtain strains yields errors obeying the scaling relationship $\epsilon_{\mathrm{RMS}} \propto v_{\mathrm{RMS}} / h_{\text {sub }}$. From the data in Fig. 3, the scaling constant is found to equal 1 ; the agreement between the prediction and the actual strain error is good, demonstrating that, for affine deformations, strain error can be ascribed solely to displacement error.

Because displacement values are correlated over a length scale roughly equal to the subset size, strain error can be minimized by choosing a gage length for strain calculation comparable to the subset size. In practice, this is equivalent to choosing a large step size: $h_{\text {st }}>h_{\text {sub }} / 2$ [4]. An alternate approach is to select an arbitrarily small step size and subsequently filter the resulting strain data. The effect of filtering on the strain error for the uniaxial tension test at an applied strain of 0.002 is illustrated in Fig. 4. For the smallest step size, two regimes are evident. When $h_{f}<h_{\text {sub }}$, averaging has almost no effect due to the aforementioned correlation 


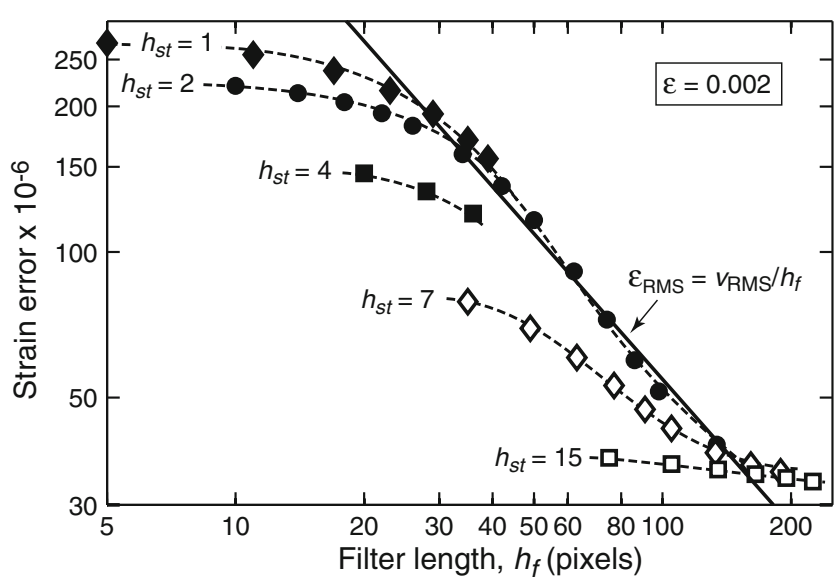

Fig. 4 Strain error vs. filter length for various step sizes $\left(h_{\text {sub }}=\right.$ 40 pixels). Strain error decreases inversely (solid line) with increasing filter length for $h_{f}>h_{\text {sub }}$. Strain error also decreases with increasing step size

of displacements and strains over a subset. In contrast, when $h_{f}>h_{\text {sub }}$, the errors follow the expected scaling relationship [12]: $\epsilon_{\mathrm{RMS}} \propto v_{\mathrm{RMS}} / h_{f}$.

Line scans of axial strain for the uniaxial tension test in Fig. 5(a) demonstrate the efficacy of filtering in reducing strain noise. Note that, when $h_{f}<h_{\text {sub }}$, strain fields oscillate with a wavelength comparable to the subset size. For large step sizes, the magnitude of strain error is diminished [4].

Differences between the DIC virtual extensometer strain and the strain measured by the laser extensometer on the specimen back-face are $\leq 50 \mu$ strain, indicating that systematic errors (strain bias) are small compared to random errors (strain standard deviation). The random errors are hundreds of $\mu$ strain before filtering. Other metrics of strain bias, e.g. the average shear strain across the specimen, are similarly small. The assumption made at the outset of the analysisthat strain bias is negligible compared to strain standard deviation-is therefore validated.

Strain gradients constitute an additional source of error, both because a secant line approximation is employed to compute displacement derivatives and because filtering is utilized to reduce noise. A Taylor series analysis proves to be insightful. Suppose that $\epsilon_{y y}$ is to be determined at a point $y_{0}$. The displacements in the neighborhood of $y=y_{0}$ can be expressed as

$v(y)=v\left(y_{0}\right)+v^{\prime}\left(y_{0}\right)\left(y-y_{0}\right)+\frac{1}{2} v^{\prime \prime}\left(y_{0}\right)\left(y-y_{0}\right)^{2}$

and the corresponding strain, computed using (firstorder) forward differences, is

$\epsilon_{y y}(y)=v^{\prime}\left(y_{0}\right)+\frac{1}{2} v^{\prime \prime}\left(y_{0}\right)\left(y-y_{0}\right)$
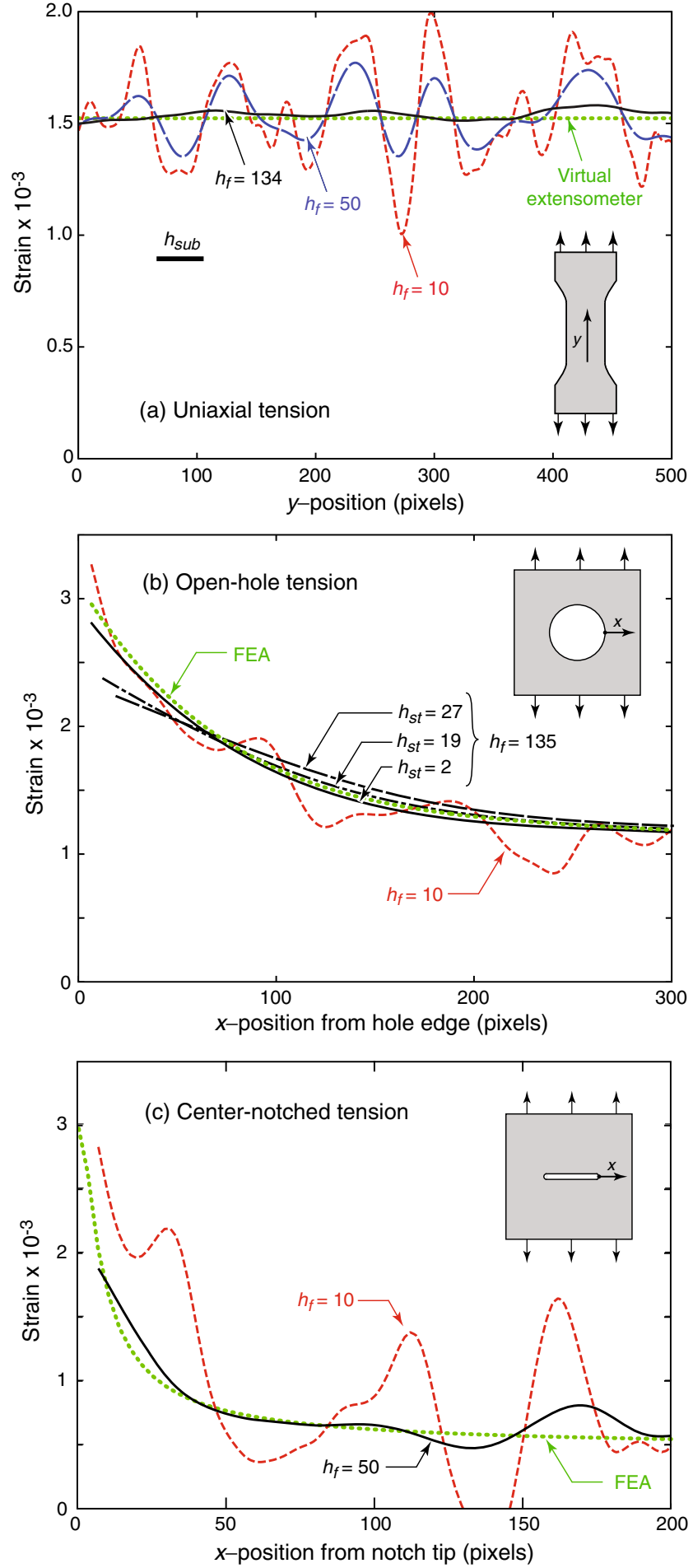

Fig. 5 (a) Line scans of strain $\left(\epsilon_{y y}\right)$ for uniaxial tension test $\left(h_{\mathrm{st}}=\right.$ 2 pixels, $h_{\text {sub }}=40$ pixels) for various filter lengths. Strain oscillations occur over a wavelength comparable to the subset size. (b) Line scans of strain $\left(\epsilon_{y y}\right)$ for open hole tension test $\left(h_{\mathrm{st}}=2\right.$ pixels, $h_{\text {sub }}=40$ pixels $)$ for minimal filtering $\left(h_{f}=10\right.$ pixels $)$ and for constant filter length ( $h_{f}=135$ pixels). Also shown for comparison is the distribution calculated by FEA. (c) Corresponding line scans for the center-notched specimen $\left(h_{\mathrm{st}}=2\right.$ pixels, $h_{\mathrm{sub}}=$ 20 pixels) 
Since the distance between adjacent nodal points is $h_{\mathrm{st}}$, the difference between the computed strain (from equation (6)) and the actual strain, $v^{\prime}\left(y_{0}\right)$, (i.e. the strain error) becomes

$\Delta \epsilon_{y y}\left(y_{0}\right)=\frac{1}{2} v^{\prime \prime}\left(y_{0}\right) h_{\mathrm{st}}$

That is, strain error is proportional to both the strain gradient and the step size. An equivalent statement is that large step sizes lead to a loss in spatial resolution of strain measurements [4]. Filtering has a similar effect. A Taylor series analysis of a uniformly weighted filter yields

$\Delta \epsilon_{y y}\left(y_{0}\right)=\frac{1}{4} v^{\prime \prime}\left(y_{0}\right) h_{f}$

for filters that are centered on the edge of a specimen (e.g. the edge of a hole or notch) and

$\Delta \epsilon_{y y}\left(y_{0}\right)=\frac{1}{24} v^{\prime \prime \prime}\left(y_{0}\right) h_{f}^{2}$

for filters lying away from a specimen edge. The deleterious effects of strain gradients and higher-order derivatives within a filter length are manifest in these relations.

The preceding relationships are confirmed by the results of the open-hole tension test. Figure 5(b) illustrates the salient trends. Line scans of strain, $\epsilon_{y y}$, along the sample mid-plane are compared to the FEA prediction at an applied stress of $100 \mathrm{MPa}$. For small filter lengths and step sizes, numerical differentiation noise is not attenuated and the strains exhibit large-amplitude oscillations (identical to those seen in the uniaxial tension test). Conversely, for large filter lengths and step sizes, strain estimates deviate from those predicted, both with increasing step size (at a constant filter length) and with increasing filter length (at a constant step size). The magnitude of the strain errors (several hundred $\mu$ strain) is large compared to the expected strain bias. At the hole edge, for step sizes large enough to reduce strain variability from numerical differentiation, the errors are well described by

$\epsilon_{\mathrm{RMS}}=0.5 \frac{d \epsilon_{y y}}{d x} h_{\mathrm{st}}+0.07 \frac{d \epsilon_{y y}}{d x} h_{f}$

The strain derivative terms were calculated using the FEA results. The first term on the right side of equation (10) is the same as that in equation (7). The coefficient $(0.07)$ of the second term differs from that $(0.25)$ obtained from the Taylor series analysis. The discrepancy is due to the fact that the Taylor series analysis employs uniformly-weighted filtering while the DIC strain calculation uses Gaussian-weighted filtering. The latter provides superior results in the presence of strain gradients.

The previous results can be utilized to generate a 'map' of acceptable parameters (magnification, subset size, filter size) for a DIC experiment on a specimen with a strain concentrator. The assumptions used to construct the map are as follows. First, the step size is chosen to be a small fraction of the subset size: $h_{\mathrm{st}}=h_{\mathrm{sub}} / 10$. Second, speckle size is assumed to be 'optimal': that is, the speckle pattern is reasonably oversampled so that $4<h_{\mathrm{sp}}<9$ pixels [2,17]. With these assumptions, the displacement error (in pixels) should be insensitive to magnification and speckle size and controlled mainly by subset size [17]. The functional form of the relationship is obtaining by fitting the data in Fig. 2(b). (Note that the magnitude of the displacement error should be confirmed by performing rigid body experiments before mechanical testing.) Third, the conservative assumption is made that strain errors arising from strain gradients and displacement noise are additive. Then, the minimum magnification needed to resolve a known strain gradient to a prescribed error tolerance can be calculated using equation (10) and relations expressed by Fig. 4. An example is shown in Fig. 6, using an error tolerance of $300 \mu$ strain. For an applied stress of $100 \mathrm{MPa}$, the resulting strain gradient is $10^{-6} / \mu \mathrm{m}$, which is indicated by the dashed line (the magnification in the open-hole tension experiment being 41 pixels $/ \mathrm{mm}$ ). Evidently, only a narrow range of subset size and filter length combinations yields acceptable strain data. One such combination is illustrated in Fig. 5(b).

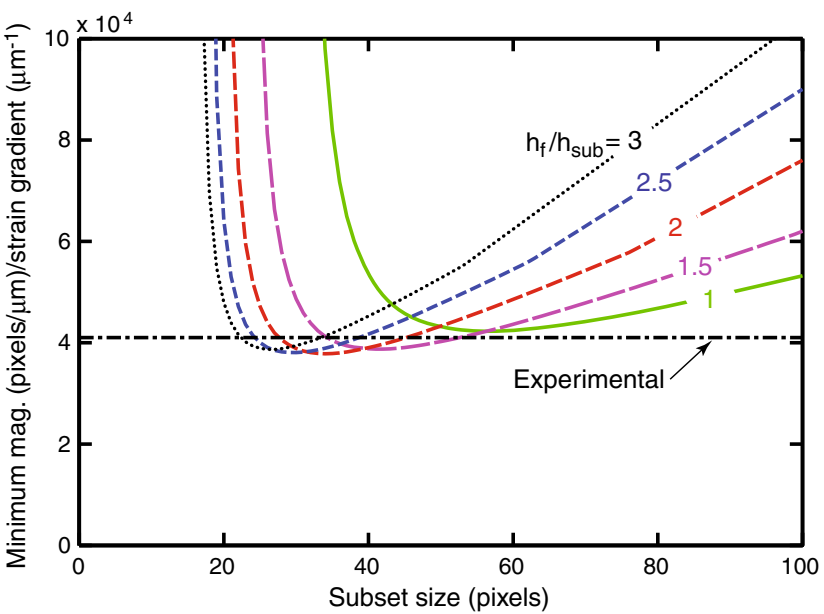

Fig. 6 Effects of subset size and filter length on the minimum magnification/strain gradient required to achieve a specified strain error (300 $\mu$ strain) in a DIC experiment 
In the center-notched geometry, the strain gradient is about an order of magnitude larger. The map indicates that, to within a reasonable strain error, DIC parameters cannot be chosen to comply with the concurrent requirements of capturing the strain gradient near the notch and mitigating the numerical differentiation noise. Line scans in Fig. 5(c) confirm this result. The gradient can be captured only by employing a higher magnification. This would also require use of smaller speckles.

These results are a direct consequence of the relationships between the length scales associated with the structural features and the DIC analysis $\left(h_{\text {sub }}, h_{f}\right)$. Specifically, in the present case, the gage length for strain computation must be significantly smaller than the notch root radius, which governs the length scale for strain decay. However, to attenuate numerical differentiation noise, the strain computation must be performed over an area large compared to the subset size, which must encompass several speckles and many more pixels. While parameters can be chosen for the open-hole and uniaxial tension tests to satisfy these opposing requirements, no such selection is possible for the center-notched test.

To summarize, errors are minimized by choosing a speckle pattern and DIC parameters in accordance with the following guidelines.

(i) Deformations within a subset should be affine; that is, the strain gradients must be small.

(ii) The subset size, speckle size, and camera magnification should be chosen to obtain full correlation
Fig. 7 (a) A representative sample of the woven fabric and (b) topographical map of $\mathrm{SiC} / \mathrm{SiC}$ composite (at the same magnification). Crossover points and flat segments can be discerned from the changes in height. (c) Tensile stress-strain response and the points (A,B,C,D) corresponding to the strain field in (d) as well as the strain and displacement fields in Fig. 8

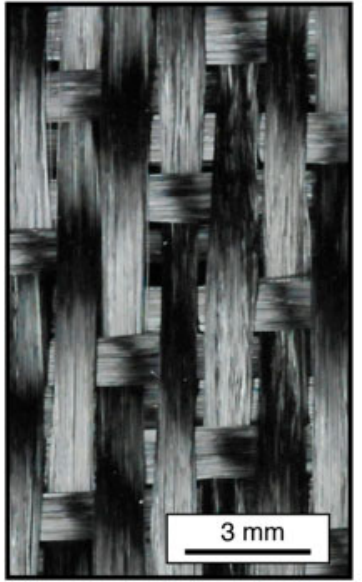

(a)

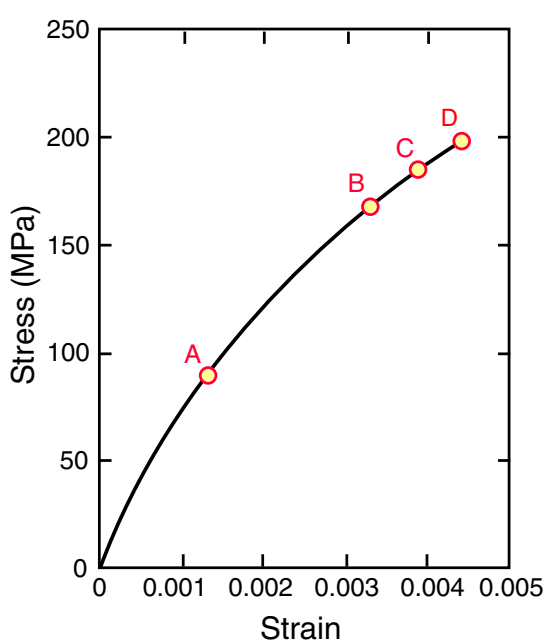

(c)

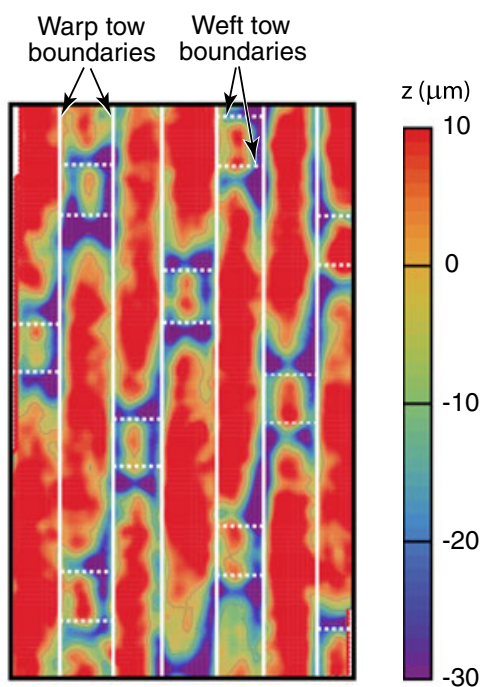

(b)

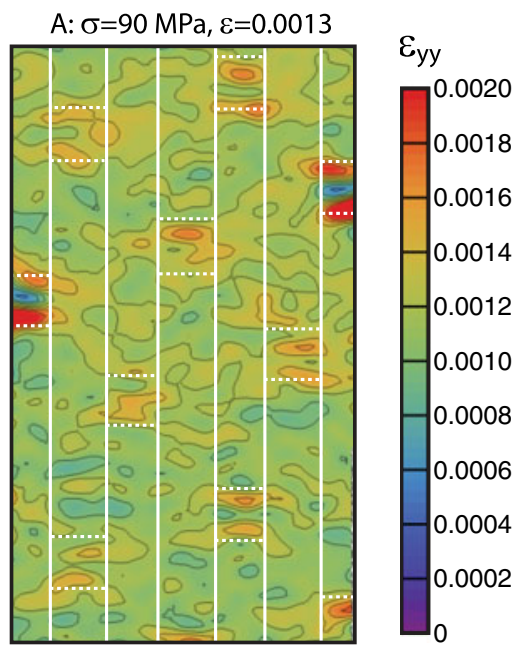

(d) 
as well as the requisite displacement accuracy. The rules of thumb $h_{\mathrm{sp}}>3$ pixels and $h_{\mathrm{sub}}>3$. $h_{\text {sp }}$ provide a useful start.

(iii) The subset size should be sufficiently small that the desired spatial resolution is achieved.

(iv) Step and filter sizes should be selected to preserve real strain gradients arising from material or structural features while simultaneously minimizing noise due to numerical differentiation.

In some cases, the parameter selection process is over-constrained. This can occur if the error tolerances are low, the area of interest is large, or the length scales for strain variation are small. Error analysis provides useful insights in selecting parameters that yield the best compromise between these competing objectives.

\section{Case Study: SiC/SiC Composite}

\section{Experimental Details}

Strain variations associated with the fiber weave in a ceramic composite under uniaxial tension were probed using DIC measurements. Samples were lasermachined from a $\mathrm{SiC} / \mathrm{SiC}$ composite comprising six layers of 2-D woven fiber (eight-harness satin weave). The specimen were machined so that the tensile direction was aligned with one set of fiber tows. The specimens had dog-bone geometry with a gauge length of $25 \mathrm{~mm}$ and gauge width of $8.5 \mathrm{~mm}$. The airbrush technique was used for speckling. Fiberglass tabs were bonded to the sample ends to facilitate uniform load transfer from the grips to the sample. The testing machine and the DIC equipment were identical to those used for the test on
Fig. 8 Strain (top) and displacement fields (bottom) at three stress levels. The white lines demarcate the tow boundaries, established in Fig. 7. Circled features are examples of cracks emanating from tow crossovers. The crack with the $x-y$ coordinate system is the one analyzed in Fig. 10. The black lines in the displacement fields are displacement contours, which are separated by $0.5 \mu \mathrm{m}$
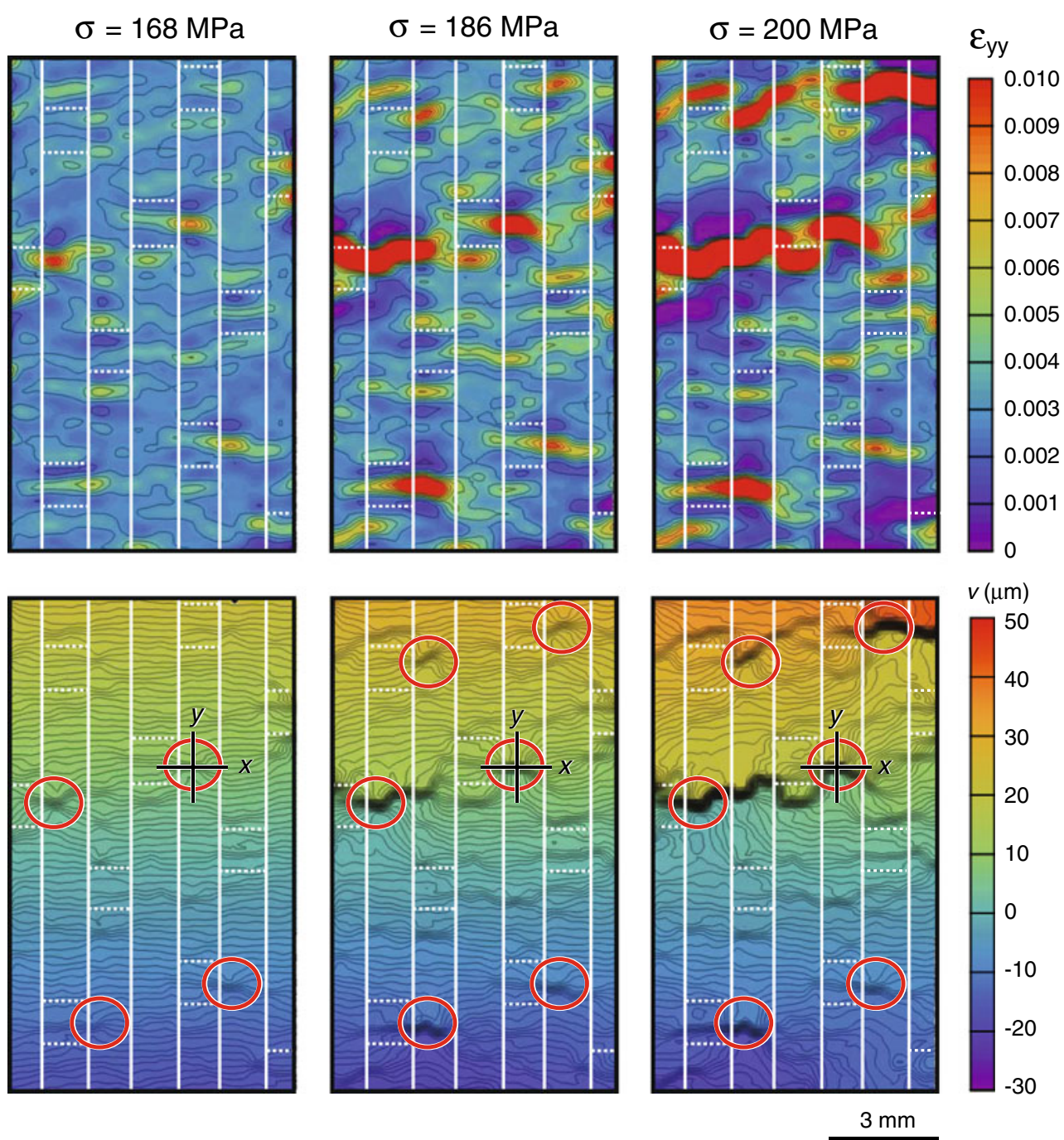
the Al specimens. A laser extensometer was used to measure strain on the back-face of the specimen over a gauge length of $25 \mathrm{~mm}$.

In the test orientations employed, surface fibers reside within either long, relatively-flat segments of longitudinal tows or short segments of transverse tows, each passing over a single longitudinal tow (Fig. 7(a)). Because the matrix conforms to the undulations of the underlying tows, crossovers and flat segments can be discerned from the topographical map generated by DIC (Fig. 7(b)).

DIC parameters were chosen to satisfy the following requirements: (i) a spatial resolution at a sub-tow length scale; (ii) full correlation across the entire sample; and (iii) a field of view that encompasses the entire specimen width. The last requirement yields an upper bound on magnification. The magnification selected for both experiments, 138 pixels $/ \mathrm{mm}$ (attained using a lens focal length of $180 \mathrm{~mm}$ ), was sufficiently high to achieve adequate spatial resolution while remaining below the upper bound. At this magnification, the area within the field of view was $12 \mathrm{~mm} \times 8.5 \mathrm{~mm}$, and $h_{\mathrm{sp}} \approx 9.5$ pixels. A compromise between the first two requirements was achieved by selecting the smallest subset size that yielded full correlation $\left(h_{\text {sub }}=51\right.$ pixels $)$. This choice implies a spatial resolution of $0.36 \mathrm{~mm}$, or roughly $30 \%$ of the tow width.

Due to the insensitivity of displacement error to strain, a good estimate of this error for low levels of applied strain $(<0.01)$ is that obtained at nominally zero strain. As stated previously, this quantity is computed by analyzing two sequential images taken before loading. The resulting estimate of displacement error is 0.013 pixels and the (unfiltered) strain error is 255 $\mu$ strain (comparable to values obtained from tests on the aluminum alloy). Since the strain noise was unacceptably large in comparison to the strain variations being measured, strains were filtered over a gage length larger than the subset size but significantly smaller than the tow width $\left(h_{f}=75\right.$ pixels $<h_{\text {tow }}=160$ pixels, $h_{\mathrm{st}}=$ 5 pixels). Based on the trends in Fig. 4, the resulting strain error induced by numerical differentiation is estimated to be approximately $100 \mu$ strain. (Note that, since $\epsilon_{\mathrm{RMS}} \approx \epsilon_{\mathrm{SD}}, \pm 2 \epsilon_{\mathrm{RMS}}$ gives a $95 \%$ confidence interval). Because strain gradients are unknown a priori, other error quantities cannot be estimated. The results
Fig. 9 (a) Line scans of strain $\left(\epsilon_{y y}\right)$ along nominally flat segments of longitudinal tows, at an applied strain of $1.4 \times 10^{-3}$ (about $30 \%$ of the tensile failure strain). The strain is not statistically different from the extensometer strain (dashed line). (b) Line scans of strain and (c) z-profiles along crossover points, at the same applied strain. Strain elevations of about $30 \%$ of the applied strain are found in the center of the transverse tow. (d) Locations of line scans (flat segments and crossover points) within the fiber architecture
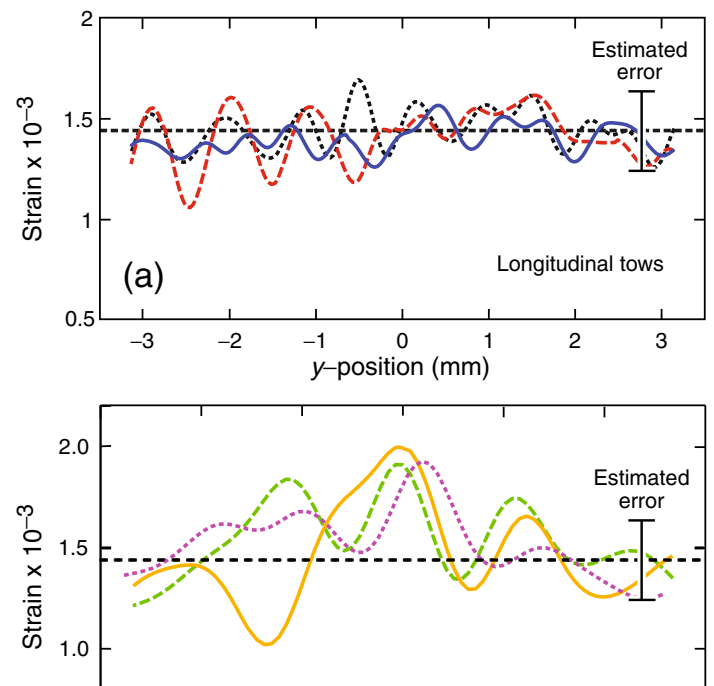

(b)
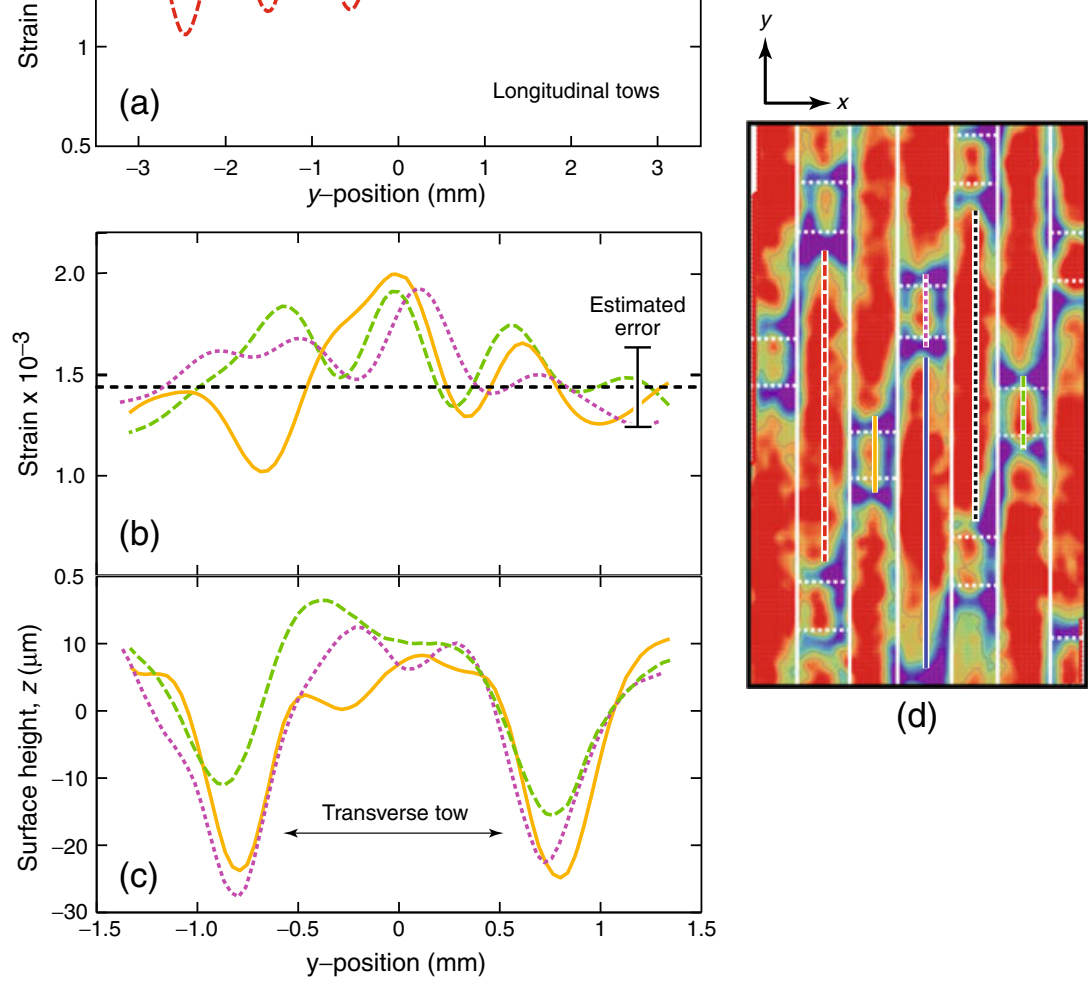

(d) 
presented below show that strain gradients can indeed become unacceptably high for accurate strain determination, especially in the vicinity of matrix cracks.

\section{Results and Discussion}

Representative results from tension tests are shown in Figs. 8, 9, and 10. At small strains (roughly half of the tensile failure strain), the strain fields are reasonably uniform (a result expected on the basis of the satin nature of the fiber weave), with one notable exception. Whereas the axial strains within the long segments of the longitudinal tows are uniform over most of their length, strain concentrations arise in locations where the transverse tows cross over the longitudinal tows. These features are illustrated by the line scans plotted in Fig. 9. Strain elevations can be rationalized on the basis of straightening of axial tows at crossover points [29]; in contrast, within nominally flat
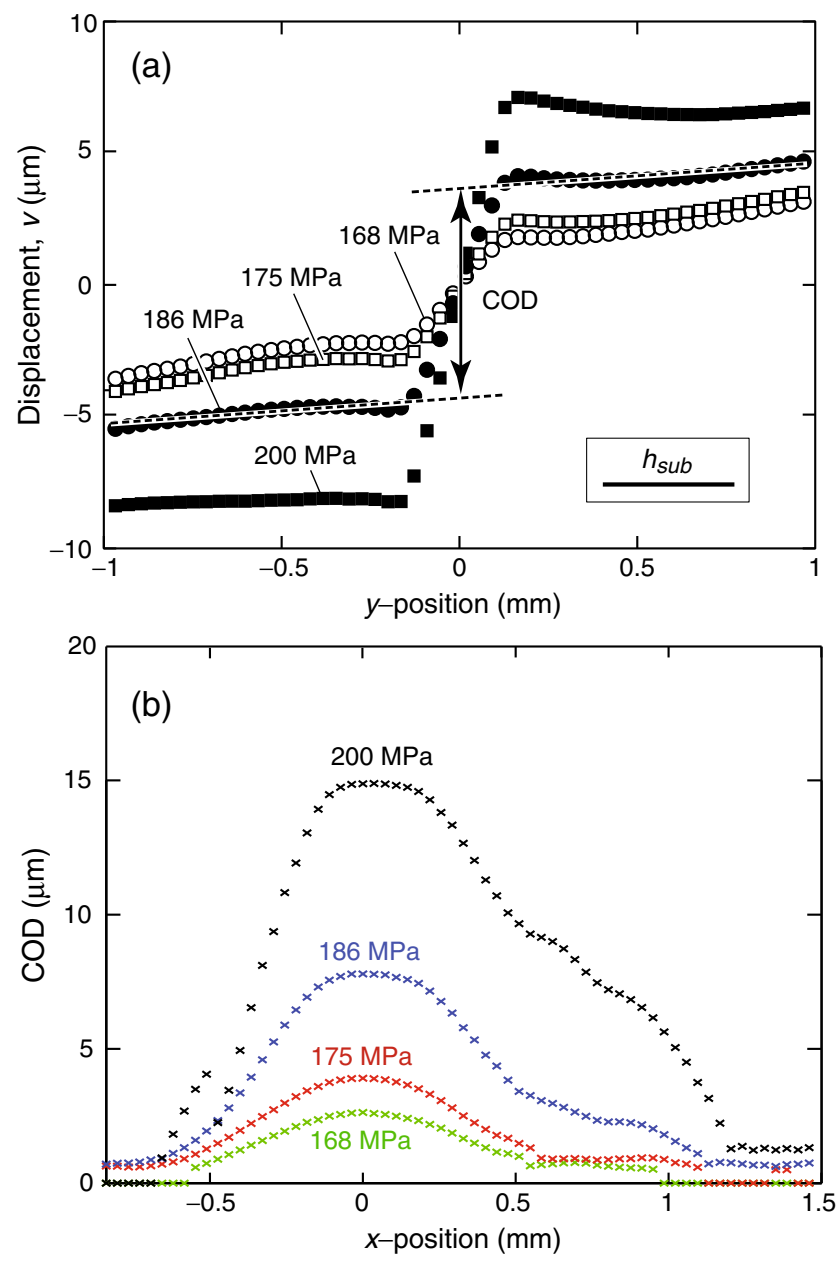

Fig. 10 (a) Demonstration of procedure used to ascertain crack opening displacement (COD). (b) COD profiles for the crack highlighted in Fig. 8 at several stress levels tow segments, undulations in the thickness direction are small and hence the strain variations are similarly small.

At larger strains $(>0.002)$, the strain concentrations subsequently 'bleed' into the adjacent longitudinal tows. Approaching the composite failure strain (0.004), strains in excess of 0.02 are seemingly attained in these tows. Upon closer examination of the displacement data, it becomes apparent that these regions are actually cracks, each producing an axial displacement discontinuity. The computed strains are therefore not true material strains. Furthermore, since these strains are essentially equal to the ratio of the local crack opening displacement (COD) to the subset size, the computed values are highly sensitive to the selection of the DIC analysis parameters.

Therefore, displacements (not strains) should be used to assess the evolution of damage within the composite. Displacement maps corresponding to the strain maps are shown in Fig. 8. In addition to identifying the crack locations, the displacement data are used to compute the COD profiles of the cracks. This is accomplished in two steps (Fig. 10(a)). First, the crack center is identified by locating the point at which the (apparent) displacement gradient along a line that straddles the crack reaches its maximum. Then, displacement data along this line are fit to a piecewise linear relationship of the form:

$v=\left\{\begin{array}{l}C_{1}\left(y-y_{\mathrm{cen}}\right)+C_{2} y_{\min } \leq y \leq y_{\mathrm{cen}}-0.67 h_{\mathrm{sub}} \\ C_{3}\left(y-y_{\mathrm{cen}}\right)+C_{4} y_{\mathrm{cen}}+0.67 h_{\mathrm{sub}} \leq y \leq y_{\max }\end{array}\right.$

where $y$ and $v$ are the displacement and position, respectively, in the direction of crack opening, and $C_{i}$ are fitting constants. This equation assumes firstorder displacements on either side of the crack. Since displacement data may be unreliable very close to the crack plane, data residing within two-thirds of a subset size from the crack center are excluded from the fitting procedure. This requirement is represented by the inequalities on the right side of equation (11). From these fits, the displacement step and hence the COD becomes $v_{\mathrm{COD}}=\left|C_{4}-C_{2}\right|$. A threshold of $0.5 \mu \mathrm{m}$ is used as a minimum value to constitute the presence of a crack. The procedure is repeated along each line passing perpendicular to the subject crack to produce the entire COD profile. Iterating at varying stress levels provides information on crack evolution. Note that crack locations can be determined much less accurately than crack opening displacements; the accuracy of the former measurement is comparable to the displacement spatial resolution (i.e. subset size), while that of the 
Fig. 11 Post-mortem micrography of the CMC tensile sample. (a) Section plane with overlaid displacement field showing crack locations, (b) Optical and (c) Electron micrographs of large central crack, and (d) Electron micrograph of small crack. Red arrows denote fiber breaks. Blue arrows denote cracks in the paint

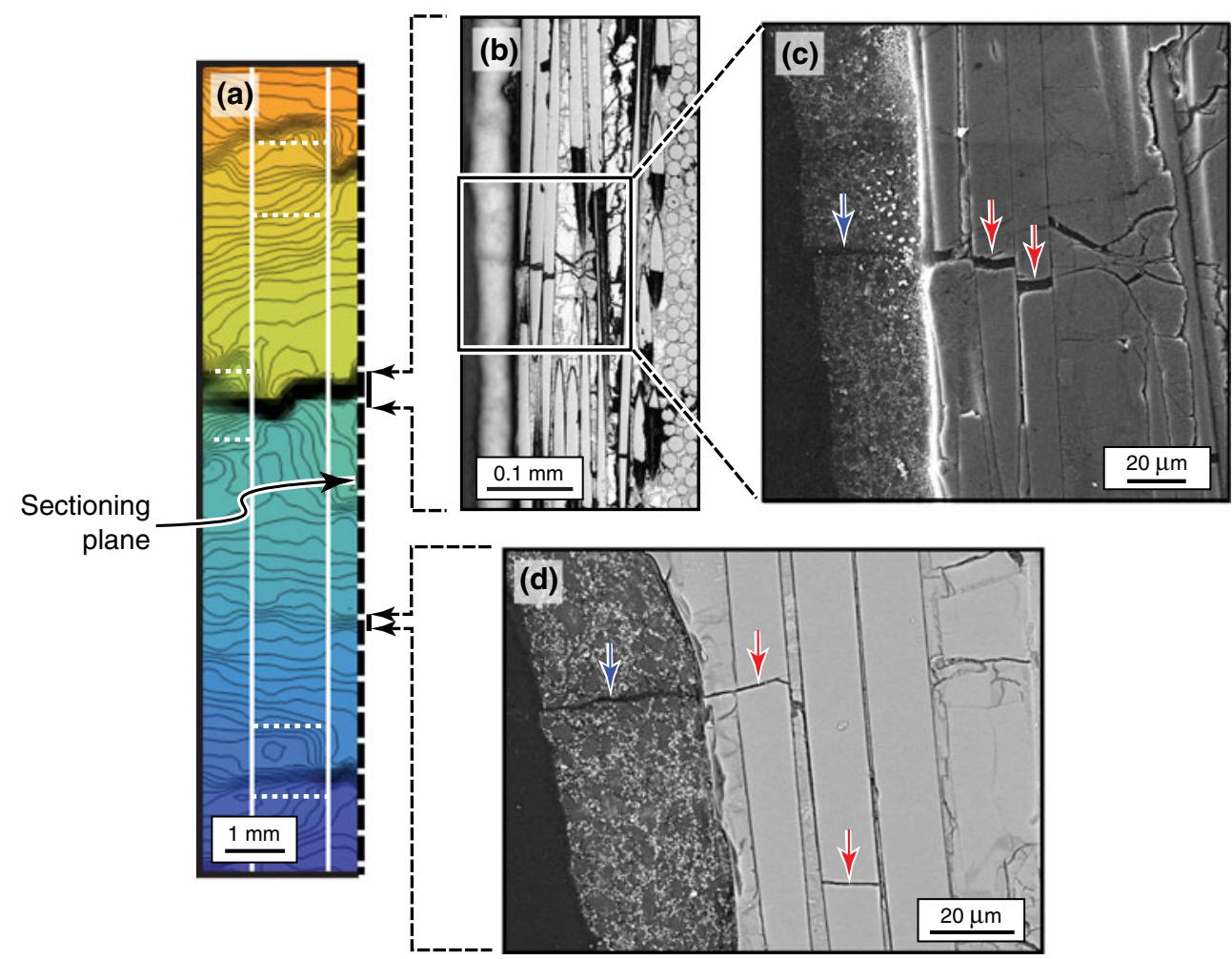

latter is comparable to the displacement error. If strain fields are instead used to detect cracks, the resolution of the measurement is related to the strain spatial resolution (i.e. filter length). This resolution is significantly lower than the displacement spatial resolution if a large filter length is employed. Efforts to determine crack locations with high fidelity are also complicated by bridging of cracks by the paint used in the speckle pattern.

COD profiles of one such crack (indicated on Fig. 8) are plotted in Fig. 10(b). At stresses slightly above that for crack nucleation, the central region of the crack exhibits an approximately elliptical profile, consistent with the prediction for a weak-bridging scenario of a through crack. The profiles near the tips diverge from the elliptical shape, presumably because of influences of neighboring cracks as well as the underlying tow architecture. It may also be a consequence of a more complex crack front in the through-thickness direction. Approaching the ultimate tensile strength, cracks begin to link. This is manifested as non-zero crack opening displacements at the outer boundaries of the data in Fig. 10(b). Current efforts are focused on extracting pertinent bridging traction laws [30] from the displacement profiles.

Post-mortem sectioning and micrography confirms the locations of cracks detected by DIC analysis (Fig. 11). That is, each displacement discontinuity captured by the DIC (Fig. 11(a)) corresponds to a transverse crack (Fig. 11(b), (c), and (d)). Each crack comprises breaks through several fibers within the surface $0^{\circ}$ tow. The DIC analysis also agrees with micrography on the relative opening displacements of different cracks.

\section{Concluding Remarks}

Digital image correlation is capable of measuring full-field displacements and strains with accuracy and spatial resolution unparalleled by strain gages and extensometers. It is especially well-suited for measuring pre-cracking strain distributions and post-cracking damage evolution in fiber composites. However, design of DIC experiments that yield high-fidelity results can be complex, primarily because numerous camera, correlation, and post-processing parameters must be selected simultaneously. These parameters can be categorized into two groups: those that must be chosen before an experiment commences (speckle pattern, camera magnification) and those that can be selected after the experiment concludes (subset size, step size, filter length). The present study shows that the optimal results are obtained when even the latter parameters are judiciously chosen prior to the test, to ensure that the requisite fidelity is indeed achievable. 
By analyzing the results of mechanical tests on an aluminum alloy in various test configurations, analytic relations for displacement and strain error have been developed and validated. Each implies a tradeoff. Higher camera magnifications entail not only higher spatial resolution of displacements but also a more limited field of view. The speckle size must be small enough so that each subset has sufficient unique information and large enough so that at least three pixels exist within each speckle. Larger subset sizes reduce displacement error at the expense of spatial resolution. Larger step sizes and filter lengths sacrifice the ability to resolve strain gradients for attenuation of strain noise. Analytic relations that capture these qualitative statements have also been provided.

With appropriate analysis parameters, digital image correlation can capture the evolution of strain within ceramic composites. The present study has illustrated that strain concentrations exist at tow crossovers even in composites with seemingly 'flat' woven fabrics. At higher stresses, the strain concentrations lead to the formation of cracks in adjacent longitudinal tows that ultimately cause rupture. In these cases, the DIC results are clearly more meaningful when presented in terms of displacements: strains calculated near a crack being non-physical in nature. A procedure to discern the locations of cracks as well as their opening displacement profiles has been presented. DIC measurements are expected to provide crucial data for calibration of high-fidelity models of the crack bridging process and composite failure.

Acknowledgements This work was supported by the Pratt \& Whitney Center of Excellence at the University of California, Santa Barbara (monitored by Douglas Berczik), and the US AFOSR (Ali Sayir) and NASA (Anthony Calomino) under the National Hypersonics Science Center for Materials and Structures (AFOSR Prime Contract No. FA9550-09-1-0477 to Teledyne Scientific and Sub-contract No. B9U538772 to UCSB). The authors gratefully acknowledge the assistance of Renaud Rinaldi with the finite element analysis.

\section{References}

1. Sutton M, McNeill S, Helm J, Chao Y (2000) Advances in two-dimensional and three-dimensional computer vision. Photomechanics 77:323-372

2. Sutton MA, Orteu J-J, Schreier HW (2009) Image correlation for shape, motion, and deformation measurements. Springer

3. Schreier HW, Sutton MA (2002) Systematic errors in digital image correlation due to undermatched subset shape functions. Exp Mech 42(3):303

4. Ke X-D, Schreier HW, Sutton MA, Wang YQ (2011) Error assessment in stereo-based deformation measurements, Part II: experimental validation of uncertainty and bias estimates. Exp Mech 51(4):423-441
5. Cox BN, Flanagan G (1997) Handbook of analytical methods for textile composites. NASA Contractor Report 4750

6. Novak MD, Zok FW (2011) High-temperature materials testing with full-field strain measurement: experimental design and practice. Rev Sci Instrum 82(11):115101

7. Bisagni C, Walters C (2008) Experimental investigation of the damage propagation in composite specimens under biaxial loading. Compos Struct 85(4):293-310

8. Kazemahvazi S, Kiele J, Zenkert D (2010) Tensile strength of UD-composite laminates with multiple holes. Compos Sci Tech 70(8):1280-1287

9. Lagattu F, Brillaud J, Lafarie-Frenot M-C (2004) High strain gradient measurements by using digital image correlation technique. Mater Charact 53(1):17-28

10. Fuchs PF, Major Z (2010) Experimental determination of cohesive zone models for epoxy composites. Exp Mech 51(5):779-786

11. Ramault C, Makris A, Van Hemelrijck D, Lamkanfi E, Van Paepegem WS (2010) Comparison of different techniques for strain monitoring of a biaxially loaded cruciform specimen. Strain 47(S2):210-217

12. Orteu J-J, Cutard T, Garcia D, Cailleux E, Robert L (2007) Application of stereovision to the mechanical characterisation of ceramic refractories reinforced with metallic fibres. Strain 43(2):96-108

13. Pankow M, Justusson B, Salvi A, Waas AM, Yen C-F, Ghiorse S (2011) Shock response of 3D woven composites: an experimental investigation. Compos Struct 93(5):13371346

14. Daggumati S, Voet E, Van Paepegem W, Degrieck J, Xu J, Lomov SV, Verpoest I (2011) Local strain in a 5-harness satin weave composite under static tension: Part I — Experimental analysis. Compos Sci Tech 71(8):1171-1179

15. Anzelotti G, Nicoletto G, Riva E (2008) Mesomechanic strain analysis of twill-weave composite lamina under unidirectional in-plane tension. Compos Part A Appl Sci Manuf 39(8):1294-1301

16. Wang Y-Q, Sutton MA, Ke X-D, Schreier HW, Reu PL, Miller TJ (2011) On error assessment in stereo-based deformation measurements, Part I: theoretical developments for quantitative estimates. Exp Mech 51(4):405-422

17. Robert L, Nazaret F, Cutard T, Orteu JJ (2007) Use of 3$\mathrm{D}$ digital image correlation to characterize the mechanical behavior of a fiber reinforced refractory castable. Exp Mech 47(6):761-773

18. Bornert M, Brémand F, Doumalin P, Dupré J-C, Fazzini M, Grédiac M, Hild F, Mistou S, Molimard J, Orteu J-J, Robert L, Surrel Y, Vacher P, Wattrisse B (2009) Assessment of digital image correlation measurement errors: methodology and results. Exp Mech 49(3):353-370

19. Avril S, Bonnet M, Bretelle A-S, Grédiac M, Hild F, Ienny P, Latourte F, Lemosse D, Pagano S, Pagnacco E, Pierron F (2008) Overview of identification methods of mechanical parameters based on full-field measurements. Exp Mech 48(4):381-402

20. Knauss WG, Huang Y, Chasiotis I (2003) Mechanical measurements at the micron and nanometer scales. Mech Mater 35(3-6):217-231

21. Haddadi H, Belhabib S (2008) Use of rigid-body motion for the investigation and estimation of the measurement errors related to digital image correlation technique. Opt Lasers Eng 46(2):185-196

22. Schreier HW, Braasch JR, Sutton MA (2000) Systematic errors in digital image correlation caused by intensity interpolation. Opt Eng 39(11):2915-2921 
23. Vic-3D (2007) ®Software. Correlated Solutions Incorporated, Columbia, SC. http://www.correlatedsolutions.com

24. Nicoletto G, Anzelotti G, Riva E (2009) Mesoscopic strain fields in woven composites: experiments vs. finite element modeling. Opt Lasers Eng 47(3-4):352-359

25. Rubin DM (2004) A simple autocorrelation algorithm for determining grain size from digital images of sediment. J Sediment Res 74(1):160-165

26. Lecompte D, Smits A, Bossuyt S, Sol H, Vantomme J, Van Hemelrijck D, Habraken AM (2006) Quality assessment of speckle patterns for digital image correlation. Opt Lasers Eng 44(11):1132-1145
27. Rasband WS (1997-2011) ImageJ, US National Institutes of Health, Bethesda, Maryland, USA. http://imagej.nih.gov/ij/

28. Sutton MA, Yan J, Deng X, Cheng C-S, Zavattieri P (2007) Three-dimensional digital image correlation to quantify deformation and crack-opening displacement in ductile aluminum under mixed-mode I/III loading. Opt Eng 46(5): $1-16$

29. González C, Llorca J (2005) Stiffness of a curved beam subjected to axial load and large displacements. Int J Solids Struct 42(5-6):1537-1545

30. Bao G, Suo Z (1992) Remarks on crack-bridging concepts. Appl Mech Rev 45(8):355-366 